\title{
High-Temperature Dynamics of the Anisotropic Heisenberg Chain Studied by Moment Methods
}

\author{
U. Brandt and J. Stolze \\ Institut für Physik, Universität Dortmund, Federal Republic of Germany \\ Received April 3, 1986
}

\begin{abstract}
The dynamics of the anisotropic spin-1/2 nearest-neighbour Heisenberg chain is studied at infinite temperature. Low-order coefficients of the short-time expansions are computed for spin-spin and energy-density-energy-density correlation functions for cyclical as well as for open-ended chains. The commutator algebra necessary to generate these coefficients may be performed by a computer. The series obtained for the spin correlation function (up to order $t^{14}$ for a bulk spin and up to order $t^{18}$ for a boundary spin) and for the energy density correlation function are the longest ones available up to now. The coefficients are used to construct rigorous upper and lower bounds to autocorrelation functions and near-neighbour correlation functions.
\end{abstract}

\section{Introduction}

Ever since the introduction of powerful magnetic resonance techniques [1] there has been considerable interest in studying the spin dynamics of simple model systems in order to understand and classify the phenomena found experimentally. The spin- $1 / 2$ anisotropic Heisenberg model is a rather general system encompassing several important special cases, some of which may be treated exactly. The dynamics of the full model is not known exactly, not even in one dimension. Recently however, Roldan, McCoy, and Perk [2] (henceforth abbreviated RMP) used moment techniques to derive impressively accurate estimates for spin autocorrelation functions of the one-dimensional version of this model. The model is specified by the Hamiltonian

$H=-\frac{1}{2} \sum_{l}\left(J_{x} \sigma_{l}^{x} \sigma_{l+1}^{x}+J_{y} \sigma_{l}^{y} \sigma_{l+1}^{y}+J_{z} \sigma_{l}^{z} \sigma_{l+1}^{z}\right)$

where $\sigma_{i}^{i}(i=x, y, z)$ denote the usual Pauli matrices (with eigenvalues \pm 1 ) representing the spin at site $l$ of the chain. The $J_{i}$ are coupling constants. The objects of interest are the infinite-temperature spin correlation functions

$$
\begin{aligned}
& c^{i}(n, t):=\left\langle\sigma_{0}^{i}(t) \sigma_{n}^{i}(0)\right\rangle_{T \rightarrow \infty} \\
& =\lim _{N \rightarrow \infty} 2^{-N} \operatorname{Tr}\left(\mathrm{e}^{i H t} \sigma_{0}^{i} \mathrm{e}^{-i H t} \sigma_{n}^{i}\right)
\end{aligned}
$$

( $N$ denotes the number of spins in the chain.) The coefficients $M_{2 l}^{i}(n)$ in the power series expansion

$c^{i}(n, t)=\sum_{l=0}^{\infty} \frac{(-1)^{l}}{2 l !} M_{2 l}^{i}(n) t^{2 l}$

may be related to the moments of a probability density (see Sect. II) for the special case $n=0$. (We shall, however, generally refer to these coefficients as "moments".) They may be calculated by evaluating repeated commutators of $H$ and $\sigma_{0}^{i}$.

We have extended the method used by RMP by calculating the required moments with the help of a computer, rather than performing the tedious commutator algebra by hand [3]. Without excessive use of computer time or storage capacity, we were able to calculate two more non-vanishing moments than given by RMP, thus improving considerably the quality of the bounds for the autocorrelation functions $c^{i}(0, t)$. We used a procedure of "resolving" the moments with respect to a complete set of plane wave states in order to improve the quality of the bounds. This procedure enabled us to derive also bounds to near-neighbour correlation functions $c^{i}(n, t)$ for a chain with cyclical boundary conditions. For open boundary conditions we studied boundary effects on the autocorrelation function. In this case, more moments may be calculated, because the number of terms of each moment is smaller due to the boundary. 
Apart from the spin-spin correlation function (1.2), we also studied the energy-density-energy-density correlation function

$c^{e}(n, t):=\left\langle e_{0}(t) e_{n}(0)\right\rangle_{T \rightarrow \infty}$

where the "energy density operator" $e_{i}$ is defined by

$e_{i}:=J_{x} \sigma_{i}^{x} \sigma_{i+1}^{x}+J_{y} \sigma_{i}^{y} \sigma_{i+1}^{y}+J_{z} \sigma_{i}^{z} \sigma_{i+1}^{z}$.

Obviously the correlation function (I.4) may be treated in a way entirely analogous to (I.2), but due to the more complicated structure of the operators involved, the moments consist of many more terms. Accordingly, one obtains generally one moment less than in the case of the spin correlation function for roughly the same amount of labour. Our reason to study also the energy-density-energy-density correlation function is the fact that the total energy is a conserved quantity, whereas the total magnetization is conserved only for $J_{x}=J_{y}$. It is of some interest to study whether conserved and non-conserved quantities differ from each other in their dynamics, especially in their respective tendencies to show diffusion-like behaviour.

In Sect. II we describe briefly the techniques which were used to calculate the moments $M_{2 l}^{i}(n)$ and the bounds to correlation functions. Section III displays some exact results for the special case $J_{x}$ $=J_{y}, J_{z}=0$, and Sect. IV is devoted to the discussion of our numerical results.

\section{Calculation of Moments and Bounds}

In this section we briefly sketch how the moments $M_{2 l}^{i}$ in (I.3) and the analogous moments in an expansion of the energy-density-energy-density correlation function may be computed numerically and how bounds to various correlation functions may be derived once the moments are known.

Expanding the exponentials in the correlation function (1.2), we obtain the following formula involving $2 l$ nested commutators:

$M_{2 l}^{z}(n)=\lim _{N \rightarrow \infty} 2^{-N} \operatorname{Tr}\left\{\left[H,\left[H, \ldots\left[H, \sigma_{0}^{z}\right] \ldots\right]\right] \sigma_{n}^{z}\right\}$

(we shall restrict ourselves to the case $i=z$ from now on, as the correlation functions $c^{x}$ and $c^{y}$ may be obtained from $c^{z}$ by an appropriate permutation of the coupling constants.) As observed by RMP, the $2 l$-fold repeated commutator in (II.1) may be split up into two $l$-fold iterated commutators which are completely identical, apart from a shift by $n$ sites:

$M_{2 l}^{z}(n)=(-1)^{l} \lim _{H \rightarrow \infty} 2^{-N} \operatorname{Tr}\left\{\left[H,\left[H, \ldots\left[H, \sigma_{0}^{z}\right] \ldots\right]\right]\right.$

$\left.\cdot\left[H,\left[H, \ldots\left[H, \sigma_{n}^{z}\right] \ldots\right]\right]\right\}$.
Obviously (II.2) may be evaluated much more efficiently than (II.1). The $l$-fold iterated commutator of $\sigma_{0}^{z}$ and $H$ in (II.2) is a sum of products of spin operators corresponding to (at most) $l+1$ consecutive sites in the region $(-l, \ldots, l)$ of the chain. The evaluation of (II.2) was completely done by computer. We limited the order $2 l$ of the moments calculated in such a way that the sum of all coefficients in $M_{2 l}^{z}(0)$ could be treated by 32 bit integer arithmetics. This limit is of course by no means fundamental. Storage capacity and computing time did not cause any problems. The results for $M_{2 l}^{z}(n)$ $(l \leqq 7)$ may be found in Table 1 . For $l \leqq 5$ they are identical to those given by RMP.

The same algorithm may of course also be used to treat an open-ended spin chain instead of the cyclical system considered up to now, in order to study boundary effects. The moments of the autocorrelation function of a spin near the boundary consist of much less terms than those of a "bulk" spin. This enables us to calculate the moments up to $l=9$ for the spin right at the boundary of the system and up to $l=8$ for the second spin. The results for $M_{2 l}^{z}(0)$ for the first spin are listed in Table 2. For the second and third spins we only give the results for the isotropic case $J_{x}=J_{y}=J_{x}$ in Table 3 ; interested readers may, however, obtain a more complete table on request.

As already mentioned in the preceding section, we also calculated the moments of the energy-density-energy-density correlation function in an analogous way. The results may be found in Table 4 for the isotropic case; again, the full results are available on request.

We now turn to the question of obtaining bounds to the correlation functions from the moments calculated up to now. As this is a well-known (and solved) problem, we shall only repeat the main points, referring the reader to the article by RMP and the literature [4-6] quoted there.

We introduce the temporal Fourier transform of the spin autocorrelation function

$\hat{c}^{z}(0, \omega):=\int_{-\infty}^{\infty} \mathrm{d} t \mathrm{e}^{\mathrm{i} \omega t} c^{z}(0, t)$.

This function is non-negative, and $\hat{c}^{z}(0, \omega) / 2 \pi$ is a normalized probability density, the moments of which are given by

$$
\begin{aligned}
& \left\langle\omega^{v}\right\rangle:=\frac{1}{2 \pi} \int_{-\infty}^{\infty} \mathrm{d} \omega \omega^{v} \hat{c}^{z}(0, \omega) \\
& =\left\{\begin{array}{ll}
0 & (v=2 l+1) \\
M_{2 l}^{z}(0) & (v=2 l)
\end{array} \quad(l=0,1, \ldots),\right.
\end{aligned}
$$


Table 1. The coefficients $M_{27}^{z}(n)$ (II.1) of the spin correlation function in a cyclical chain are listed in the following form: below each combination of $2 l$ and $n$ yielding a non-vanishing coefficient, the first three entries in each row denote powers of $J_{x}, J_{y}$, and $J_{z}$, respectively, and the last entry is the coefficient corresponding to this combination of powers. Thus, for example,

$M_{4}^{2}(1)=-4 J_{x} J_{y} J_{z}^{2}-12 J_{x} J_{y}^{3}-12 J_{x}^{3} J_{y}$.

Below these entries, we give the sum of the coefficients, which is the only information necessary to specify the moments for the isotropic case $J_{x}=J_{y}=J_{z}$. These numbers are identical to the corresponding entries in Table I of [2]

\begin{tabular}{|c|c|c|c|}
\hline $2 l=2$ & $n=0$ & & \\
\hline 0 & 2 & 0 & 2 \\
\hline 2 & 0 & 0 & 2 \\
\hline Sum: & & & 4 \\
\hline $2 l=2$ & $n=1$ & & \\
\hline 1 & 1 & 0 & -2 \\
\hline Sum: & & & -2 \\
\hline $2 l=4$ & $n=0$ & & \\
\hline 0 & 2 & 2 & 4 \\
\hline 0 & 4 & 0 & 8 \\
\hline 2 & 0 & 2 & 4 \\
\hline 2 & 2 & 0 & 20 \\
\hline 4 & 0 & 0 & 8 \\
\hline Sum: & & & 44 \\
\hline $2 l=4$ & $n=1$ & & \\
\hline 1 & 1 & 2 & -4 \\
\hline 1 & 3 & 0 & -12 \\
\hline 3 & 1 & 0 & -12 \\
\hline Sum: & & & -28 \\
\hline
\end{tabular}

$2 l=4 \quad n=2$

$\begin{array}{llll}2 & 2 & 0 & 6 \\ \text { Sum: } & & & 6\end{array}$

$2 l=6 \quad n=0$

$\begin{array}{lllr}0 & 2 & 4 & 16 \\ 0 & 4 & 2 & 72 \\ 0 & 6 & 0 & 32 \\ 2 & 0 & 4 & 16 \\ 2 & 2 & 2 & 76 \\ 2 & 4 & 0 & 168 \\ 4 & 0 & 2 & 72 \\ 4 & 2 & 0 & 168 \\ 6 & 0 & 0 & 32 \\ \text { Sum: } & & & 652\end{array}$

$2 l=6 \quad n=1$

$\begin{array}{llll}1 & 1 & 4 & -16 \\ 1 & 3 & 2 & -70 \\ 1 & 5 & 0 & -64 \\ 3 & 1 & 2 & -70 \\ 3 & 3 & 0 & -172 \\ 5 & 1 & 0 & -64 \\ & & & -456\end{array}$

52

$$
2 l=6 \quad n=2
$$

$$
\begin{array}{lll}
2 & 2 & 2 \\
2 & 4 & 0 \\
4 & 2 & 0
\end{array}
$$

Sum:

150

$$
2 l=6 \quad n=3
$$

$\begin{array}{llll}3 & 3 & 0 & -20 \\ \text { Sum: } & & & -20\end{array}$

\begin{tabular}{cll}
\hline $2 l=8$ & $n=0$ & \\
0 & 2 & 6 \\
0 & 4 & 4 \\
0 & 6 & 2 \\
0 & 8 & 0 \\
2 & 0 & 6 \\
2 & 2 & 4 \\
2 & 4 & 2 \\
2 & 6 & 0 \\
4 & 0 & 4 \\
4 & 2 & 2 \\
4 & 4 & 0 \\
6 & 0 & 2 \\
6 & 2 & 0 \\
8 & 0 & 0
\end{tabular}

\section{Sum:}

11,636

$$
2 l=8 \quad n=1
$$

\begin{tabular}{llll}
1 & 1 & 6 & -64 \\
1 & 3 & 4 & -504 \\
1 & 5 & 2 & -680 \\
1 & 7 & 0 & -320 \\
3 & 1 & 4 & -504 \\
3 & 3 & 2 & $-2,112$ \\
3 & 5 & 0 & $-1,640$ \\
5 & 1 & 2 & -680 \\
5 & 3 & 0 & $-1,640$ \\
7 & 1 & 0 & -320 \\
\cline { 2 - 2 } Sum: & & & $-8,464$
\end{tabular}

$2 l=8 \quad n=2$

$\begin{array}{lll}2 & 2 & 4 \\ 2 & 4 & 2 \\ 2 & 6 & 0 \\ 4 & 2 & 2 \\ 4 & 4 & 0 \\ 6 & 2 & 0\end{array}$

Sum:

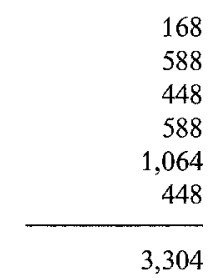

848

640

128
64

$-16$

1,824

1,152

848
1,824

2,340

640

1,152

128

504

680

504

$-2,112$

$-680$

$-1,640$

$-8,464$
$2 l=8 \quad n=3$

\begin{tabular}{cccc}
3 & 3 & 2 & -168 \\
3 & 5 & 0 & -280 \\
5 & 3 & 0 & -280 \\
Sum: & & & -728 \\
\hline
\end{tabular}

$2 l=8 \quad n=4$

44

70

Sum:

70

$$
2 l=10 \quad n=0
$$

$\begin{array}{rrr}0 & 2 & 8 \\ 0 & 4 & 6 \\ 0 & 6 & 4 \\ 0 & 8 & 2 \\ 0 & 10 & 0 \\ 2 & 0 & 8 \\ 2 & 2 & 6 \\ 2 & 4 & 4 \\ 2 & 6 & 2 \\ 2 & 8 & 0 \\ 4 & 0 & 6 \\ 4 & 2 & 4 \\ 4 & 4 & 2 \\ 4 & 6 & 0 \\ 6 & 0 & 4 \\ 6 & 2 & 2 \\ 6 & 4 & 0 \\ 8 & 0 & 2 \\ 8 & 2 & 0 \\ 10 & 0 & 0\end{array}$

256
Sum:

14,496

4,480

512

256

$-10,048$

18,024

25,048

7,040

10,496

18,024

43,760

24,200

14,496

25,048

24,200

4,480

7,040

512

242,816

$2 l=10 \quad n=1$

$\begin{array}{llll}1 & 1 & 8 & - \\ 1 & 3 & 6 & - \\ 1 & 5 & 4 & -168 \\ 1 & 7 & 2 & -5,332 \\ 1 & 9 & 0 & -5,248 \\ 3 & 1 & 6 & -3,536 \\ 3 & 3 & 4 & -31,144 \\ 3 & 5 & 2 & -32,804 \\ 3 & 7 & 0 & -12,768 \\ 5 & 1 & 4 & -5,332 \\ 5 & 3 & 2 & -32,804 \\ 5 & 5 & 0 & -24,312 \\ 7 & 1 & 2 & -5,248 \\ 7 & 3 & 0 & -12,768 \\ 9 & 1 & 0 & -1,536 \\ & & & -177.424\end{array}$


Table 1 (continued)

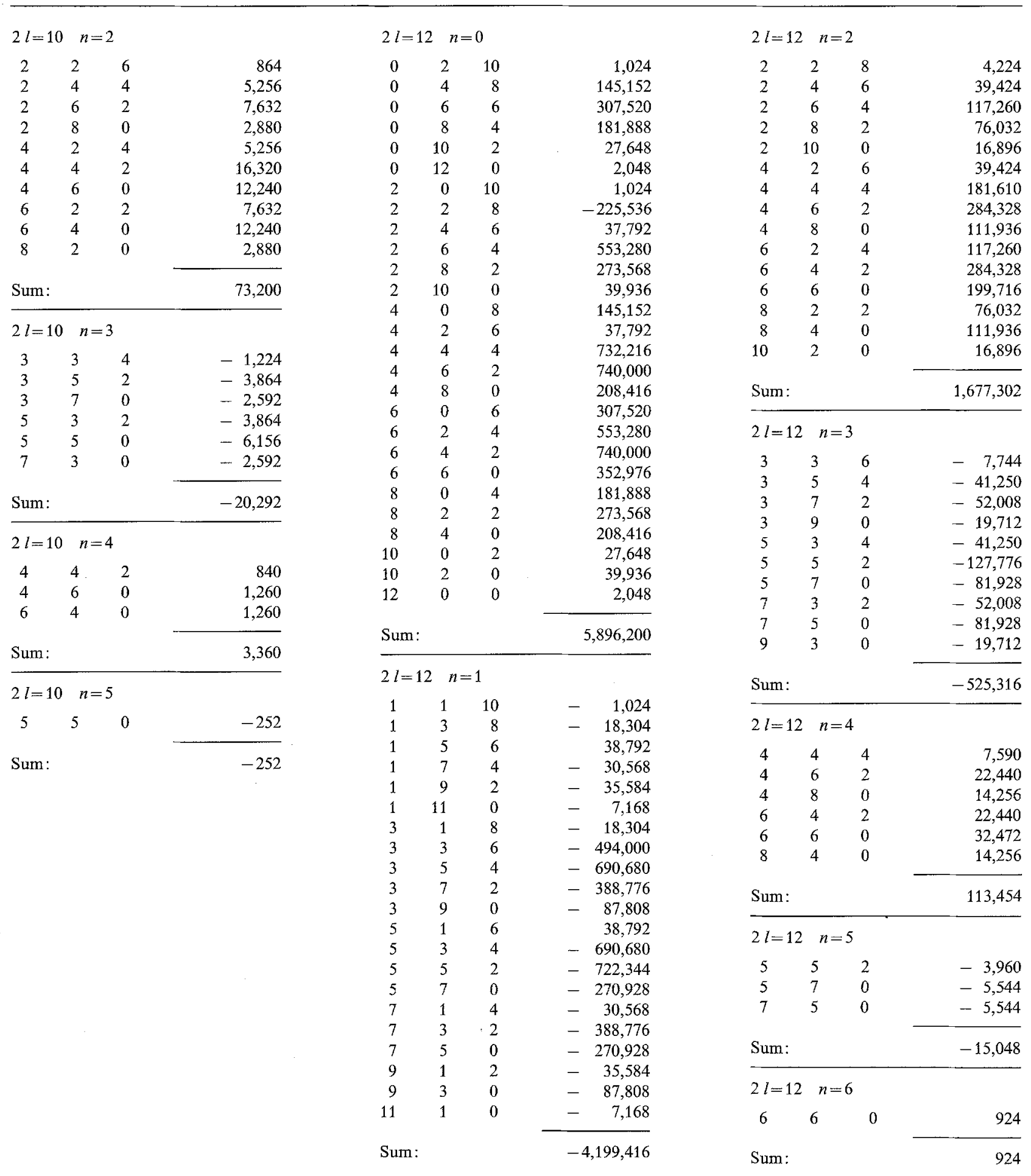


Table 1 (continued)

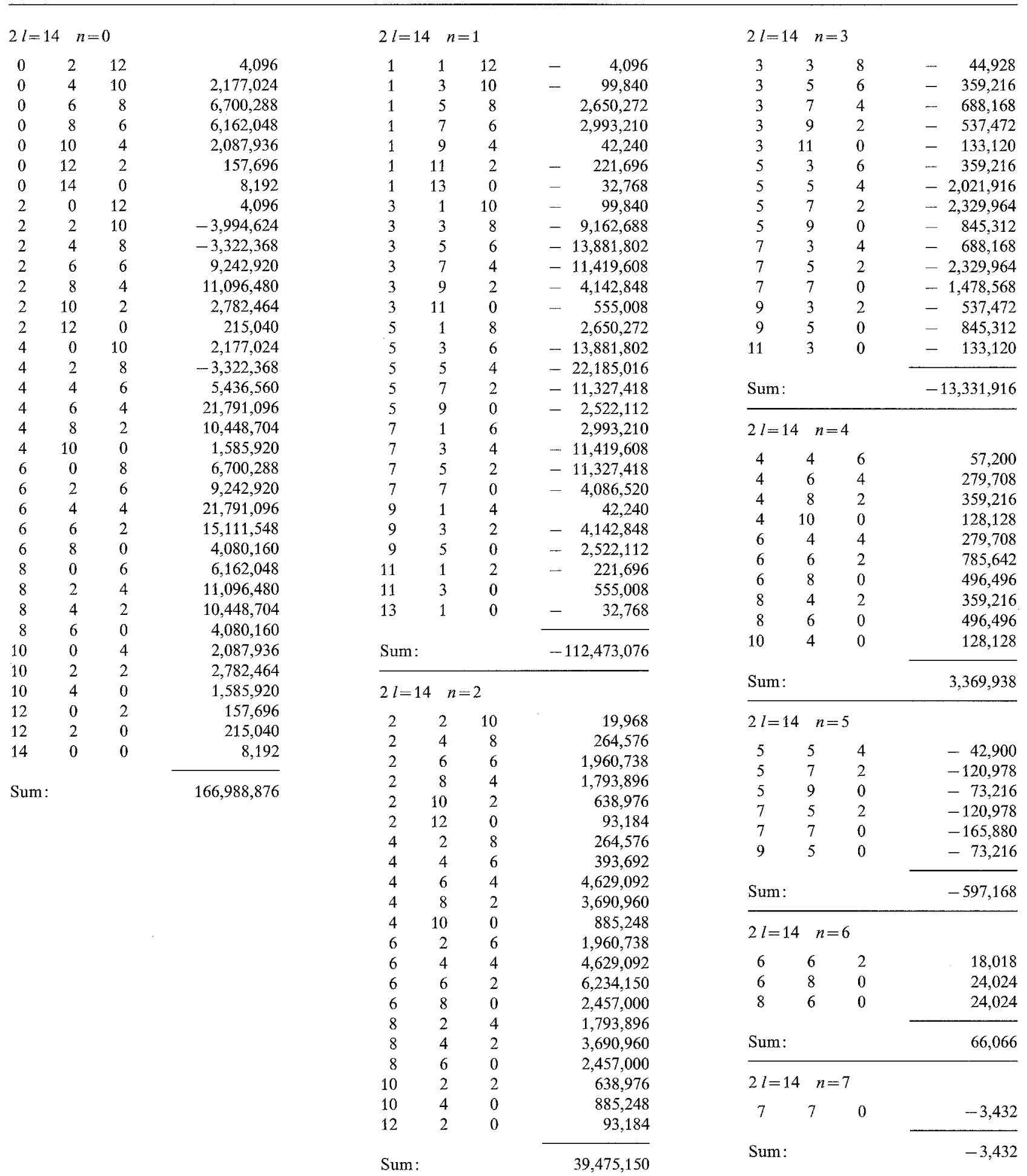


Table 2. The coefficients $M_{2 l}^{z}(0)$ (II.1) of the autocorrelation function of the first spin in an open-ended chain are listed in the same form as used in Table 1

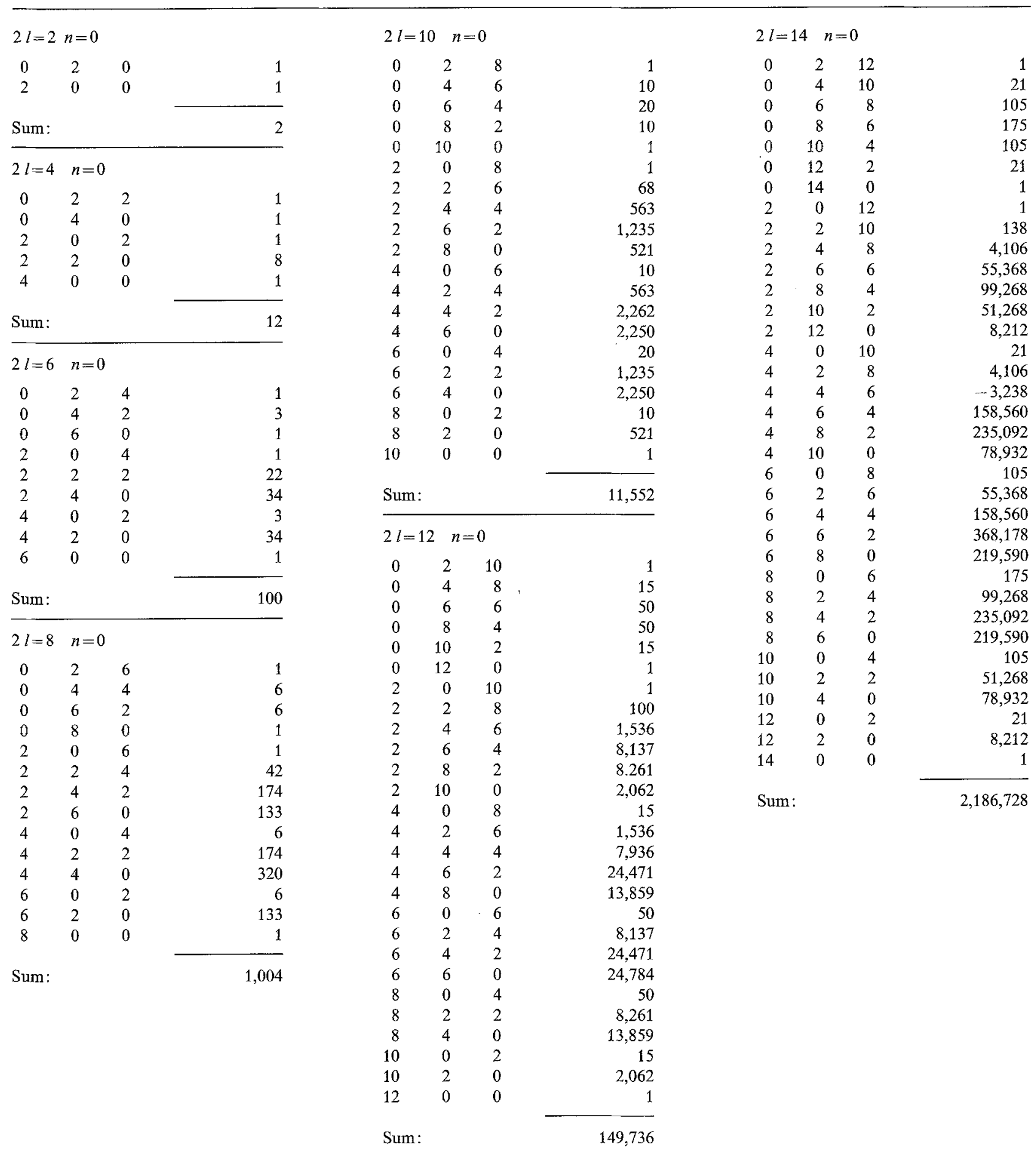


Table 2 (continued)

\begin{tabular}{|c|c|c|c|c|c|c|c|}
\hline \multicolumn{3}{|c|}{$2 l=16 \quad n=0$} & \multicolumn{5}{|c|}{$2 l=18 \quad n=0$} \\
\hline 0 & 2 & 14 & 1 & 0 & 2 & 16 & 1 \\
\hline 0 & 4 & 12 & 28 & 0 & 4 & 14 & 36 \\
\hline 0 & 6 & 10 & 196 & 0 & 6 & 12 & 336 \\
\hline 0 & 8 & 8 & 490 & 0 & 8 & 10 & 1,176 \\
\hline 0 & 10 & 6 & 490 & 0 & 10 & 8 & 1,764 \\
\hline 0 & 12 & 4 & 196 & 0 & 12 & 6 & 1,176 \\
\hline 0 & 14 & 2 & 28 & 0 & 14 & 4 & 336 \\
\hline 0 & 16 & 0 & 1 & 0 & 16 & 2 & 36 \\
\hline 2 & 0 & 14 & 1 & 0 & 18 & 0 & 1 \\
\hline 2 & 2 & 12 & 182 & 2 & 0 & 16 & 1 \\
\hline 2 & 4 & 10 & 11,908 & 2 & 2 & 14 & 232 \\
\hline 2 & 6 & 8 & 428,178 & 2 & 4 & 12 & 38,975 \\
\hline 2 & 8 & 6 & $1,195,906$ & 2 & 6 & 10 & $3,608,778$ \\
\hline 2 & 10 & 4 & 996,908 & 2 & 8 & 8 & $14,555,970$ \\
\hline 2 & 12 & 2 & 296,796 & 2 & 10 & 6 & $18,374,002$ \\
\hline 2 & 14 & 0 & 32,795 & 2 & 12 & 4 & $8,746,830$ \\
\hline 4 & 0 & 12 & 28 & 2 & 14 & 2 & $1,625,061$ \\
\hline 4 & 2 & 10 & 11,908 & 2 & 16 & 0 & 131,107 \\
\hline 4 & 4 & 8 & $-380,384$ & 4 & 0 & 14 & 36 \\
\hline 4 & 6 & 6 & 629,268 & 4 & 2 & 12 & 38,975 \\
\hline 4 & 8 & 4 & $2,871,480$ & 4 & 4 & 10 & $-5,018,340$ \\
\hline 4 & 10 & 2 & $2,017,144$ & 4 & 6 & 8 & $-1,748,846$ \\
\hline 4 & 12 & 0 & 426,152 & 4 & 8 & 6 & $33,213,582$ \\
\hline 6 & 0 & 10 & 196 & 4 & 10 & 4 & $41,168,994$ \\
\hline 6 & 2 & 8 & 428,178 & 4 & 12 & 2 & $15,827,093$ \\
\hline 6 & 4 & 6 & 629,268 & 4 & 14 & 0 & $2,212,140$ \\
\hline 6 & 6 & 4 & $3,466,648$ & 6 & 0 & 12 & 336 \\
\hline 6 & 8 & 2 & $4,693,500$ & 6 & 2 & 10 & $3,608,778$ \\
\hline 6 & 10 & 0 & $1,697,062$ & 6 & 4 & 8 & $-1,748,846$ \\
\hline 8 & 0 & 8 & 490 & 6 & 6 & 6 & $18,254,976$ \\
\hline 8 & 2 & 6 & $1,195,906$ & 6 & 8 & 4 & $73,756,664$ \\
\hline 8 & 4 & 4 & $2,871,480$ & 6 & 10 & 2 & $52,214,380$ \\
\hline 8 & 6 & 2 & $4,693,500$ & 6 & 12 & 0 & $11,923,272$ \\
\hline 8 & 8 & 0 & $2,640,640$ & 8 & 0 & 10 & 1,176 \\
\hline 10 & 0 & 6 & 490 & 8 & 2 & 8 & $14,555,970$ \\
\hline 10 & 2 & 4 & 996,908 & 8 & 4 & 6 & $33,213,582$ \\
\hline 10 & 4 & 2 & $2,017,144$ & 8 & 6 & 4 & $73,756,664$ \\
\hline 10 & 6 & 0 & $1,697,062$ & 8 & 8 & 2 & $76,025,172$ \\
\hline 12 & 0 & 4 & 196 & 8 & 10 & 0 & $26,564,556$ \\
\hline 12 & 2 & 2 & 296,796 & 10 & 0 & 8 & 1,764 \\
\hline 12 & 4 & 0 & 426,152 & 10 & 2 & 6 & $18,374,002$ \\
\hline 14 & 0 & 2 & 28 & 10 & 4 & 4 & $41,168,994$ \\
\hline 14 & 2 & 0 & 32,795 & 10 & 6 & 2 & $52,214,380$ \\
\hline \multirow[t]{2}{*}{16} & 0 & 0 & 1 & 10 & 8 & 0 & $26,564,556$ \\
\hline & & & & 12 & 0 & 6 & 1,176 \\
\hline \multirow[t]{10}{*}{ Sum: } & & & $36,324,140$ & 12 & 2 & 4 & $8,746,830$ \\
\hline & & & & 12 & 4 & 2 & $15,827,093$ \\
\hline & & & & 12 & 6 & 0 & $11,923,272$ \\
\hline & & & & 14 & 0 & 4 & 336 \\
\hline & & & & 14 & 2 & 2 & $1,625,061$ \\
\hline & & & & 14 & 4 & 0 & $2,212,140$ \\
\hline & & & & 16 & 0 & 2 & 36 \\
\hline & & & & 16 & 2 & 0 & 131,107 \\
\hline & & & & 18 & 0 & 0 & 1 \\
\hline & & & & Su & & & $693,696,880$ \\
\hline
\end{tabular}


Table 3. The coefficients $M_{2 I}^{2}(0)$ (II.1) of the autocorrelation functions of the second and third spins in an open-ended chain for the isotropic case $J_{x}=J_{y}=J_{z}$. The entries of this table correspond to the entries denoted "sum" in Tables 1 and 2

\begin{tabular}{rrr}
\hline $2 l$ & 2nd spin & 3rd spin \\
\hline 2 & 4 & 4 \\
4 & 40 & 44 \\
6 & 488 & 644 \\
8 & 6,868 & 11,036 \\
10 & 110,556 & 214,052 \\
12 & $2,039,104$ & $4,677,656$ \\
14 & $43,087,956$ & $115,829,856$ \\
16 & $1,035,393,124$ & \\
\hline
\end{tabular}

Tables $4 \mathrm{a}$ and b. The dimensionless coefficients $M_{2 l}^{e}(n) / J^{2 l+2}$ of the energy-density-energy-density correlation function for the isotropic case $J_{x}=J_{y}=J_{z}=J$

a) for a cyclical chain, general $n$, b) for the first three bonds in an open-ended chain, $n=0$

\begin{tabular}{|c|c|c|c|c|c|c|c|}
\hline$n^{2 l}$ & & 2 & 4 & 6 & 8 & 10 & 12 \\
\hline 0 & 3 & 12 & 144 & 2,472 & 51,696 & $1,232,784$ & $32,369,952$ \\
\hline 1 & & -6 & -96 & $-1,848$ & $-41,016$ & $-1,012,188$ & $-27,104,388$ \\
\hline 2 & & & 24 & 732 & 20,040 & 555,960 & $15,952,704$ \\
\hline 3 & & & & -120 & $-5,544$ & $-198,180$ & $-6,599,868$ \\
\hline 4 & & & & & 672 & 42,048 & $1,861,200$ \\
\hline 5 & & & & & & 4,032 & $-\quad 319,968$ \\
\hline 6 & & & & & & & 25,344 \\
\hline $2 l$ & & & & 1st bond & & 2nd bond & $3 \mathrm{rd}$ bond \\
\hline 0 & & & & 3 & & 3 & 3 \\
\hline 2 & & & & 6 & & 12 & 12 \\
\hline 4 & & & & 36 & & 132 & 144 \\
\hline 6 & & & & 348 & & 1,860 & 2,448 \\
\hline 8 & & & & 4,512 & & 30,888 & 49,224 \\
\hline 10 & & & & 71,604 & & 582,216 & $1,097,100$ \\
\hline 12 & & & & $1,315,224$ & & $12,134,856$ & $26,405,352$ \\
\hline 14 & & & & $26,946,852$ & & $74,683,120$ & \\
\hline
\end{tabular}

The inverse transformation corresponding to (II.3) is

$c^{z}(0, t)=\frac{1}{2 \pi} \int_{-\infty}^{\infty} \mathrm{d} \omega \cos \omega t \hat{c}^{z}(0, \omega)=\langle\cos \omega t\rangle$.

The problem of finding bounds to the autocorrelation function $c^{z}(0, t)$ is now reduced to the mathematical problem of finding the largest and smallest values of the average of the smooth function $f(\omega)$ $=\cos \omega t$ consistent with the given finite set of moments $\left\langle\omega^{v}\right\rangle \quad\left(v=0, \ldots, 2 l_{\text {max }}\right)$. Tchebycheff theory $[5,6]$ tells us that these extremal values are generated by probability densities which consist of (at most) $2 l_{\max }+1$ delta functions (and which possess the given set of moments). Platz and Gordon [4] devel- oped a linear optimization procedure for finding these probability densities. For a more restricted class of functions $f(\omega)$ (with non-vanishing $\left(2 l_{\max }\right.$ $+1)$ th derivative with respect to $\omega$ ) closer bounds may be given $[5,6]$, using the "principal representations" [5] of the given set of moments, i.e. probability densities consisting of (roughly, cf. [5] for details) $l$ delta functions [7]. Although these bounds are clearly not applicable in the case $f(\omega)=\cos \omega t$, we may use the principal representations of the given set of moments as input for the linear optimization procedure mentioned above.

Another quantity for which bounds may be derived is the spatial Fourier transform of the spin autocorrelation function (of the cyclical chain)

$$
\begin{aligned}
\tilde{c}^{z}(k, t): & =\sum_{n=-\infty}^{\infty} \mathrm{e}^{\mathrm{i} k n} c^{z}(n, t) \\
& =c^{z}(0, t)+2 \sum_{n=1}^{\infty} c^{z}(n, t) \cos k n .
\end{aligned}
$$

This is due to the positivity of the dynamic structure factor

$S^{z}(k, \omega)=\int_{-\infty}^{\infty} \mathrm{d} t \mathrm{e}^{\mathrm{i} \omega t} \tilde{c}^{z}(k, t)$

the moments of which are defined in a way analogous to (II.4) and are given by

$\tilde{M}_{2 l}^{z}(k)=M_{2 l}^{z}(0)+2 \sum_{n=1}^{\infty} M_{2 l}^{z}(n) \cos k n$.

Using the inverse transformation corresponding to (II.6):

$c^{z}(n, t)=\frac{1}{\pi} \int_{0}^{\pi} \mathrm{d} k \cos k n \tilde{c}^{z}(k, t)$

one may obtain bounds to $c^{z}(n, t)$ from the bounds to $\tilde{c}^{x}(k, t)$ by simply substituting the upper and lower bounds to the integrand in (II.9) for every $k$ and integrating (numerically) afterwards. The bounds to the near-neighbour correlation functions $c^{z}(n, t)$ (n $=1,2,3)$ turned out to be of similar quality as the bounds to $c^{z}(0, t)$ (see Figs. 4 and 7). The bounds to $c^{z}(0, t)$ obtained by this method must be better than the bounds derived from the moments (II.4) of the autocorrelation function, since more information is incorporated in them, namely the $M_{2 l}^{z}(n)$ for $n \neq 0$. Furthermore this information has been "resolved" with respect to $k$, which should be especially useful if the eigenstates of the system were superpositions of plane-wave-like elementary excitations [8]. It turned out, however, that the $k$-resolution procedure did 
not produce a drastic improvement of the bounds for this model. This indicates that the eigenstates of the system do not enjoy the special property mentioned above. The bounds to $c^{z}(n, t)$ for $n \neq 0$ may not be obtained by any other method. The properties of the energy-density-energy-density correlation function $c^{e}(n, t)$ are fully analogous to those of the spin-spin correlation function $c^{z}(n, t)$ and thus the same methods may be applied to obtain bounds.

\section{Exact Results for the $X Y$ Model}

As already stated by RMP, only very few exact results are known about the dynamics of the Hamiltonian (II.1), mainly for the case $J_{z}=0$. The most important of these results are reviewed in [2] and need not be repeated here. Because of our interest in boundary effects, we have calculated the spin autocorrelation function $c_{i}^{z}(0, t)$ for the $i$-th spin in an open-ended semi-infinite chain. We briefly sketch the calculation here, as we do not know of any treatment of this correlation function in the literature. The spin operators are first transformed to fermion operators by a Jordan-Wigner transformation [9]. In terms of these operators, the Hamiltonian (II.1) for $J_{x}=0$ now reads

$$
\begin{aligned}
H= & -\sum_{i=1}^{N-1}\left\{\left(J_{x}-J_{y}\right)\left(a_{i}^{+} a_{i+1}^{+}+a_{i+1} a_{i}\right)\right. \\
& \left.+\left(J_{x}+J_{y}\right)\left(a_{i}^{+} a_{i+1}+a_{i+1}^{+} a_{i}\right)\right\} .
\end{aligned}
$$

Here the fermion operator $a_{i}^{+}$creates a particle at site $i$ :

$|i\rangle=a_{i}^{+}|0\rangle$;

the $a$ operators fulfill the usual anticommutator relations. The Hamiltonian (III.1) is a bilinear form in the fermion operators; its diagonalization is thus straightforward. We further simplify the problem by assuming $J_{x}=J_{y}=J$. In this case, the normalized one-particle eigenstates $|v\rangle$ of (III.1) are given by

$$
\langle i \mid v\rangle=\left(\frac{2}{N+1}\right)^{1 / 2} \sin \frac{v \pi i}{N+1} \quad(v=1, \ldots, N)
$$

and the corresponding energy eigenvalues are

$\varepsilon_{\nu}=-4 J \cos \frac{\nu \pi}{N+1}$.

The Hamiltonian now reads

$$
H=-4 J \sum_{v=1}^{N} a_{\nu}^{+} a_{\nu} \cos \frac{v \pi}{N+1}
$$

where the operator $a_{v}^{+}$creates a fermion in the state $|v\rangle$ (III.3). The spin autocorrelation function $c_{i}^{z}(0, t)$ may be expressed by a fermion number autocorrelation function

$c_{i}^{z}(0, t)=\left\langle\mathrm{e}^{\mathrm{i} H t}\left(2 a_{i}^{+} a_{i}-1\right) \mathrm{e}^{-\mathrm{i} H t}\left(2 a_{i}^{+} a_{i}-1\right)\right\rangle_{T \rightarrow \infty}$.

This expression is most easily evaluated in the representation (III.3-5), using Wick's theorem. The result is (for $N \rightarrow \infty$ )

$c_{i}^{z}(0, t)=\left[J_{0}(2 J t)-(-1)^{i} J_{2 i}(2 J t)\right]^{2}$

where the $J_{n}$ are Bessel functions. The behaviour of this function is illustrated in Figs. 1a-c. The most prominent feature apart from the rapid initial decay is the echo-like structure clearly visible in the $i=2$ and $i=3$ curves in Figs. $1 \mathrm{a}$ and $1 \mathrm{~b}$. This maximum seems to be associated with the first maximum of the Bessel function $J_{2 i}$. A glance at Fig. 1c shows that there is a roughly linear relation between the distance to the chain end and the time at which the echo occurs. This indicates the "strongly non-diffusive" dynamical behaviour of the $X Y$ moddel. The occurrence of "echoes" in the spin correlation function is not restricted to the case $J_{z}=0$ as will become evident below.

The analogous correlation function $c_{i}^{x}(0, t)$ of the $X Y$ model (corresponding to $c_{i}^{z}$ for $J_{x}=J_{z}$ ) may be calculated similarly for any finite $i$; however, for increasing $i$ the calculation involves the evaluation of increasingly complicated determinants. For $i \rightarrow \infty$ these determinants may be evaluated to yield a Gaussian decay of the correlation function [10]. No pronounced echoes are visible in this case (see Fig. 9).

\section{Numerical Results and Discussion}

This section contains a discussion of the numerical results represented in the figures, and of some results not represented there.

To demonstrate the effect of the number of moments on the quality of the bounds derived from these moments, we display in Fig. 2 the autocorrelation function $c_{1}^{z}(0, t)$ of the first spin in an openended chain for the isotropic Heisenberg system, $J_{x}$ $=J_{y}=J_{z}=1$. The three pairs of bounds represented there were calculated from moments up to orders $2 l$ $=8,12$, and 18 , respectively. The bounds from moments up to order 18 clearly reveal that after the rapid initial decay for $t<1.5$ the autocorrelation function shows a much slower decay (if it decays at al1). If course the asymptotic behaviour for $t \rightarrow \infty$ cannot be inferred from any finite number of moments without further assumptions. 

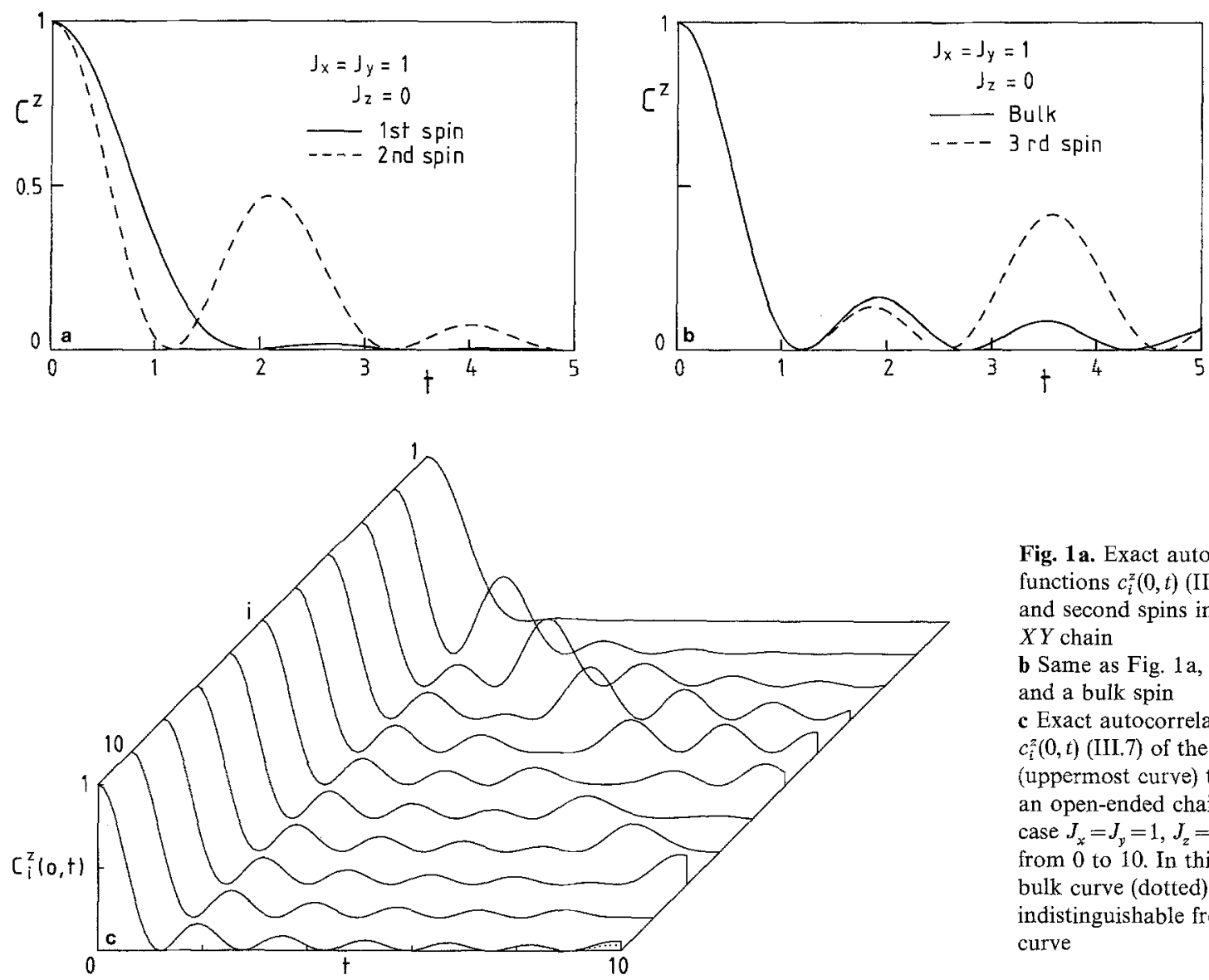

Fig. 1a. Exact autocorrelation functions $c_{i}^{z}(0, t)($ III.7) of the first and second spins in an open-ended $X Y$ chain

b Same as Fig. 1a, for the third spin and a bulk spin

c Exact autocorrelation functions $c_{i}^{z}(0, t)$ (III.7) of the spins $i=1$ (uppermost curve) through $i=11$ in an open-ended chain, for the $X Y$ case $J_{x}=J_{y}=1, J_{z}=0$. Time ranges from 0 to 10 . In this time range the bulk curve (dotted) is almost indistinguishable from the $i=11$ curve

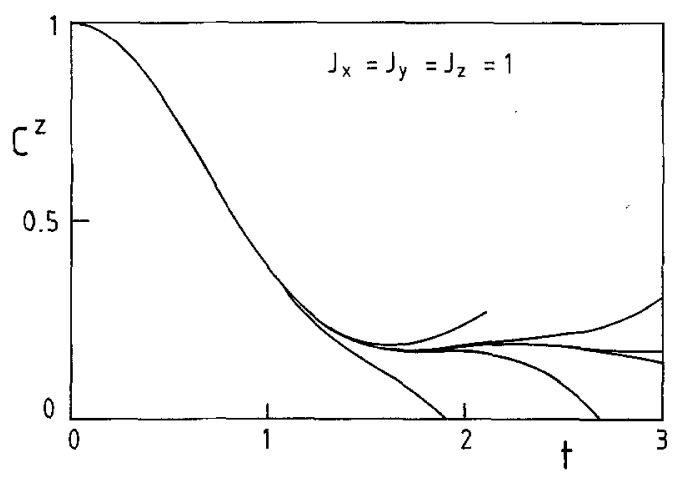

Fig. 2. Three pairs of bounds to the autocorrelation function $c_{1}^{z}(0, t)$ of the first spin in an open-ended isotropic Heisenberg system, using moments up to orders 8,12 , and 18 , respectively

As expected from (I.3), the initial decay of the spin autocorrelation function is determined by the second moment (involving only nearest neighbours of the spin considered). This is clearly visible in Figs. 1a, 3a, 6a, 8, 9: the initial decay of the first spin in an open-ended chain is slower by a factor of $2^{1 / 2}$ than the initial decay of the second spin. However, the characteristic time of this decay does not change any more, if the third spin (or a bulk spin) is considered instead of the second spin. Figure 5 indicates that the energy-density-energy-density correlation function $c^{e}$ behaves similarly.

As a matter of fact, we have not found any dramatic qualitative differences between the correlation functions $c^{e}$ and $c^{z}$; so we only show Fig. 5 as an example for the behaviour of $c^{e}$.

The "echoes" clearly exhibited in the $X Y$ case (see Fig. 1) also show up for $J_{z} \neq 0$, for example in Fig. $3 \mathrm{a}$ for the isotropic Heisenberg case (see also Fig. 5a for the corresponding echo in the energydensity-energy-density correlation function) and in Fig. 6 a for $J_{x}=J_{y}=1, J_{z}=0.5$. As a general rule the oscillatory behaviour shown for $J_{z}=0$ is damped for increasing $J_{z}$, changing to a rapid initial decay followed by a "shoulder". This indicates the presence of at least two relevant time scales in the system. The short time scale is determined by nearest-neighbour interactions, as discussed above. To gain insight into the nature of the long-time behaviour, it seems to be necessary to calculate further moments or to use entirely different methods. In all cases studied, we observed that the bounds to the bulk 

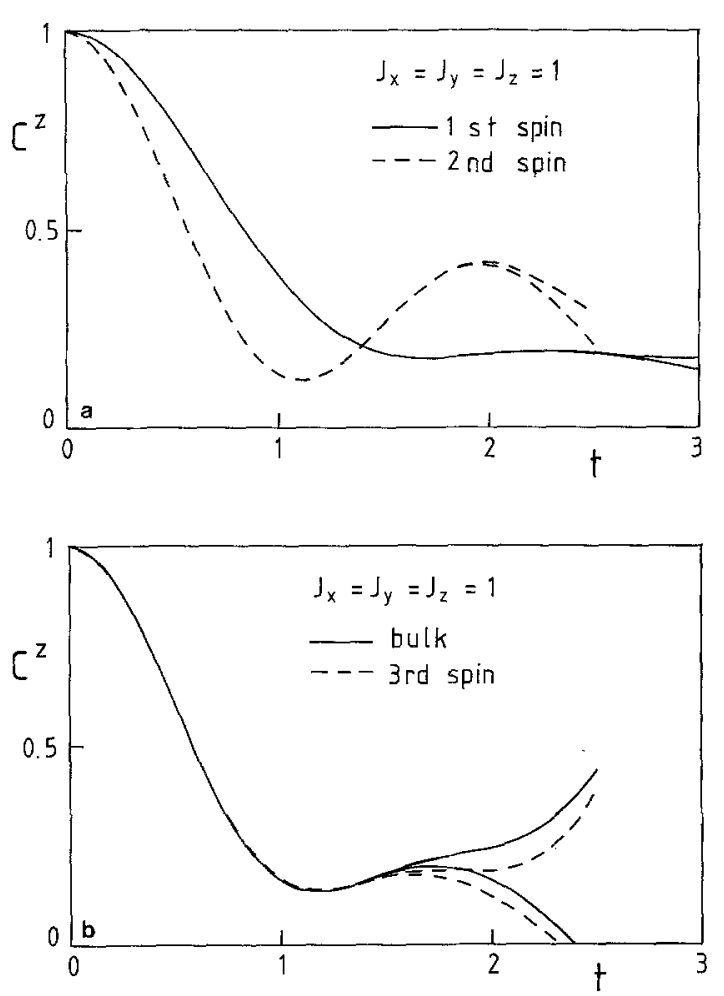

Fig. 3. a Bounds to the autocorrelation functions $c_{i}^{z}(0, t)$ of the first and second spins in an open-ended isotropic Heisenberg chain. b Same as Fig. 3a, for the third spin and a bulk spin

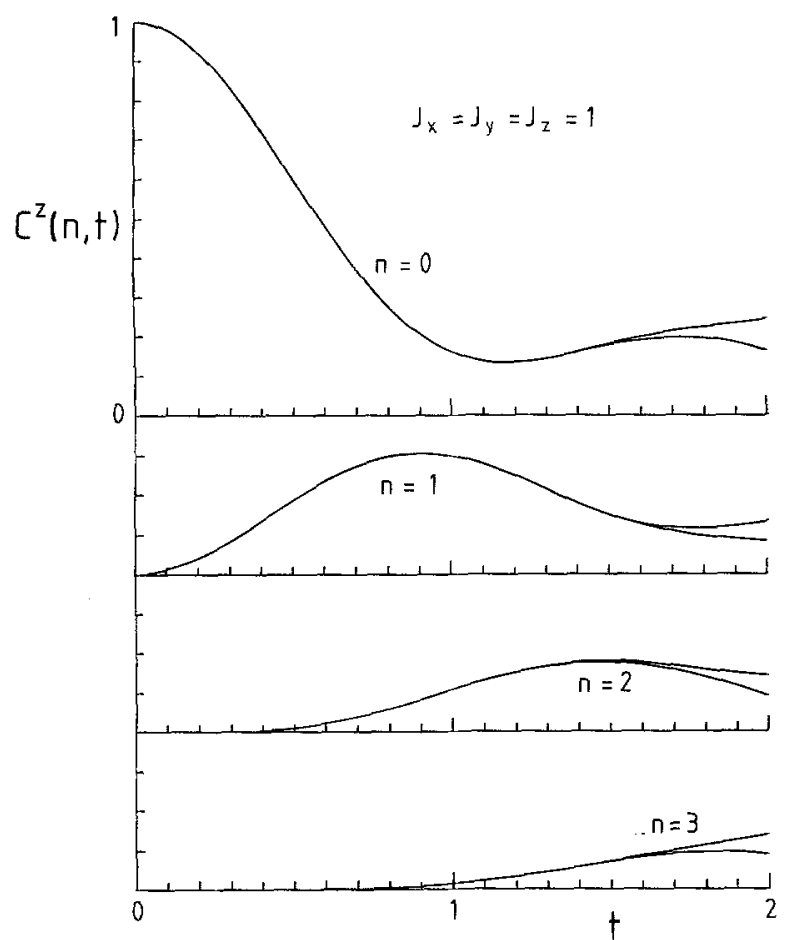

Fig. 4. Bounds to the spin autocorrelation function $c^{x}(0, t)$ and near-neighbour correlation functions $c^{z}(n, t)$ in a cyclical isotropic Heisenberg chain
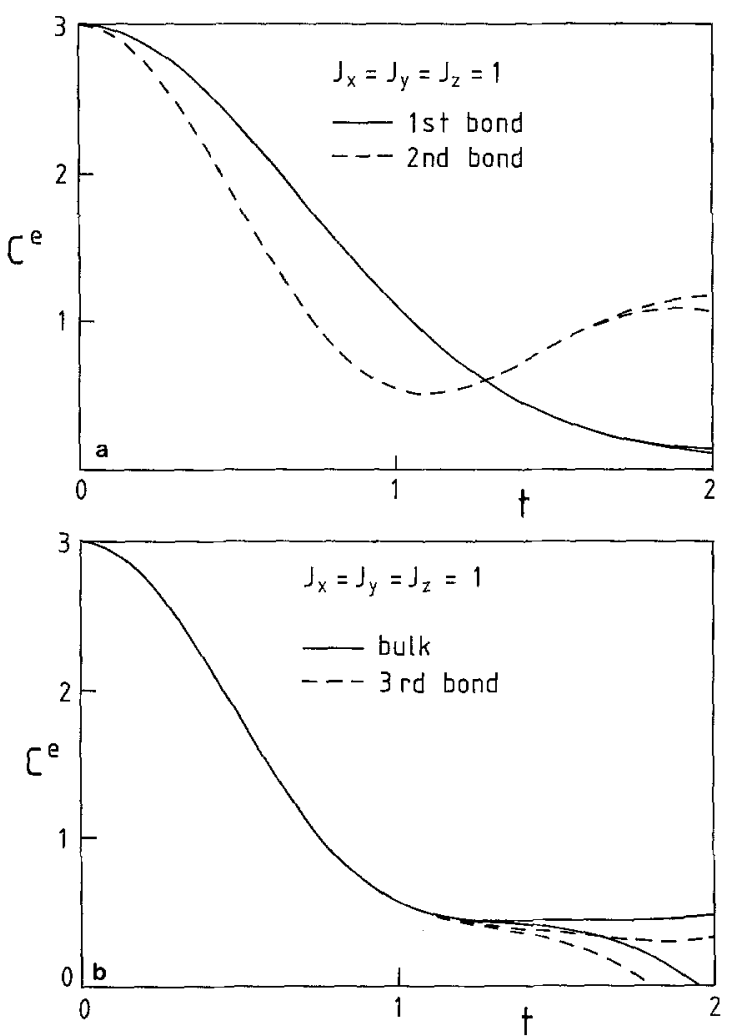

Fig. 5. a Bounds to the energy density autocorrelation functions $c_{i}^{e}(0, t)$ of the first and second bonds in an open-ended isotropic Heisenberg chain. Note that the initial value of the energy density autocorrelation function is $J_{x}^{2}+J_{y}^{2}+J_{z}^{2}$. b Same as Fig. 5 a, for the third bond and a bulk bond

spin autocorrelation function $c^{z}(0, t)$ were consistent with $c^{z}(0, t)$ being non-negative. (The same observation applies to the energy-density-energy-density correlation function.) We are, however, not aware of any proof of such a general statement. Near the boundary of an open-ended chain, $c^{z}(0, t)$ may show negative values, as displayed in Fig. 9 for $J_{x}=J_{z}=1$, $J_{y}=0$, where the bulk autocorrelation function is known to be a Gaussian [10]. Furthermore we observed from our numerical results that the bulk spin autocorrelation function $c^{z}(0, t)$ seems to be a monotonously increasing function of $J_{z}$ for $J_{x}=J_{y}$, whereas the energy density autocorrelation function does not show such behaviour.

In conclusion, we have shown that the moment approach to the calculation of dynamic spin autocorrelation functions for the anisotropic Heisenberg model suggested by RMP may be generalized to treat different (and more complicated) correlation functions. Using a computer for the calculation of the commutators makes these calculations practically feasible. The time range in which close bounds to correlations functions are available was extended well beyond the range of the initial decay, and it 

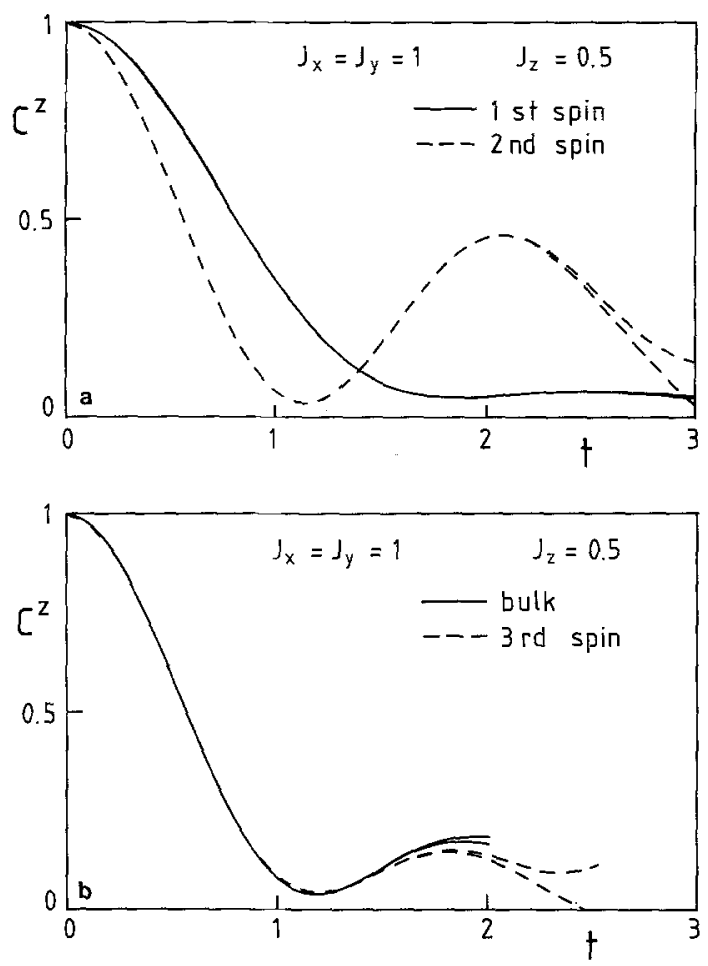

Fig. 6. a Bounds to the autocorrelation functions $c_{i}^{z}(0, t)$ of the first and second spins in an open-ended anisotropic Heisenberg chain. b Same as Fig. $6 a$, for the third spin and a bulk spin

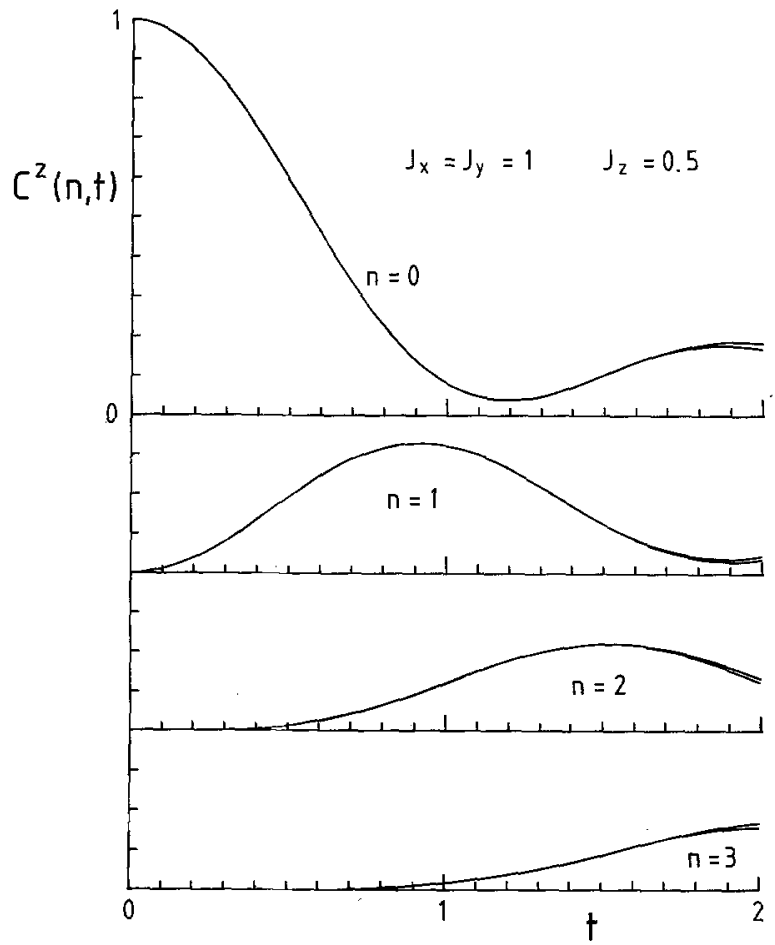

Fig. 7. Bounds to the spin autocorrelation function $c^{z}(0, t)$ and near-neighbour correlation functions $c^{z}(n, t)$ in a cyclical anisotropic Heisenberg chain

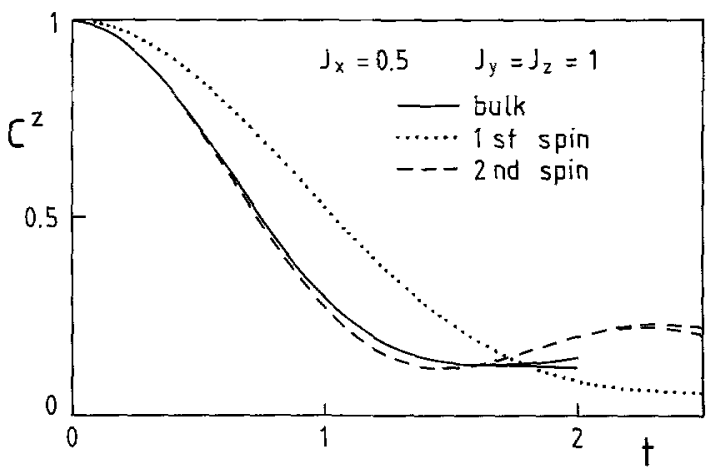

Fig. 8. Bounds to the autocorrelation functions $c_{i}^{z}(0, t)$ of the first and second spins, and of a bulk spin, in an open-ended anisotropic Heisenberg chain

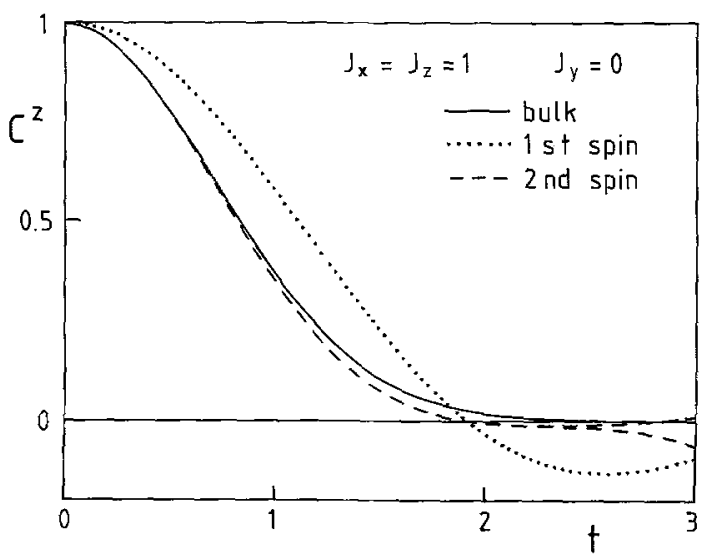

Fig. 9. Autocorrelation function $c_{i}^{z}(0, t)$ for an " $X Z$ " system, equivalent to the autocorrelation functions $c^{x}$ or $c^{y}$ of an $X Y$ system, for the first and second spins, and for a bulk spin. Curves for the first spin and the bulk spin represent exact results, for the second spin, bounds to the autocorrelation function are shown. The bulk spin autocorrelation function is a Gaussian: $c^{z}(0, t)$ $=\exp \left(-J^{2} t^{2}\right) .\left(J:=J_{x}=J_{z}\right)$

should not be difficult to compute some further moments to further extend this range. However we wish to stress once more that it remains very difficult to assess the validity of spin diffusion theory for large times from a finite number of moments.

\section{Note Added in Proof}

The correlation function (III.7) has been evaluated earlier by L.L. Gonçalves and H.B. Cruz: J. Magn. Magn. Mater. 15-18, 1067 (1980). We are grateful to Dr. J.H.H. Perk, who pointed this out to us in a letter containing also other helpful remarks.

\section{References}

1. Slichter, C.P.: Principles of magnetic resonance. In: Springer Series in Solid-State Sciences, Vol.1, 2nd Edn. Berlin, Heidelberg, New York: Springer-Verlag 1978 
2. Roldan, J.M.R., McCoy, B.M., Perk, J.H.H.: Preprint ITP-SB45, State University of New York, Stony Brook. Physica A (to be published)

3. The idea of evaluating spin commutators by computer is not new, cf. e.g. Morita, T.: J. Math. Phys. 12, 2062 (1971)

Oitmaa, J., Plischke, M., Winchester, T.A.: Phys. Rev. B29, 1321 (1984)

4. Platz, O., Gordon, R.G.: Phys. Rev. Lett. 30, 264 (1973)

5. Krein, M.G.: Am. Math. Soc. Transl., Ser. 2, 12, 1 (1959)

6. Karlin, S., Studden, W.J.: Tchebycheff Systems: with Applications in Analysis and Statistics. New York: Wiley 1966

7. These bounds are often useful in thermodynamics, for a review of applications cf. e.g. Wheeler, J.C., Gordon, R.G.: The Padé approximant in theoretical Physics. Baker, Jr., G.A., Gammel, J.L. (eds.), p. 99. New York: Academic Press 1970

8. See Brandt, U., Stolze, J.: Z. Phys. B - Condensed Matter 43, 61 (1981) for an example
9. Cf.e.g. Lieb, E., Schultz, T., Mattis, D.: Ann. Phys. (NY) 16, 407 (1961)

10. Brandt, U., Jacoby, K.: Z. Phys. B - Condensed Matter 25, $181(1976) ; 26,245(1977)$

Capel, H.W., Perk, J.H.H.: Physica 87A, 211 (1977)

Perk, J.H.H., Capel, H.W.: Physica 89A, 265 (1977); 92A, 163 (1978)

Perk, J.H.H., Capel, H.W., Siskens, Th.J.: Physica 89A, 304 (1977)

U. Brandt

J. Stolze

Institut für Physik

Universität Dortmund

Postfach 500500

D-4600 Dortmund 50

Federal Republic of Germany 